\title{
Aggressive blood pressure reduction in patients at high vascular risk: is it dangerous?
}

\section{Il trattamento antipertensivo aggressivo in pazienti ad elevato rischio vascolare è dannoso?}

\section{Fabio Angeli ${ }^{a, *}$, Gianpaolo Reboldi ${ }^{b}$, Cristina Poltronieri ${ }^{a}$, Giovanni Mazzotta c, Marta Garofoli c, Elisa Ramundo c, Alessandra Biadetti ${ }^{c}$, Paolo Verdecchia ${ }^{c}$}

\footnotetext{
a Section of Cardiology, Hospital "Media Valle del Tevere", ASL 2 Umbria, Perugia, Italy

'Department of Internal Medicine, University of Perugia, Italy

c Department of Internal Medicine, Hospital of Assisi, Italy
}

\section{KEY WORDS \\ J-curve; \\ Blood pressure goals; \\ Hypertension; \\ Treatment; \\ Cardiovascular risk.}

\begin{abstract}
Summary
Introduction: The aim of this review was to summarize the current state of evidence regarding the optimal blood pressure goals in patients with high vascular risk. In particular, this review critically addresses the issue of the "J-curve" paradox - a hypothesis indicating that low treatment-induced blood pressure values are characterized by an increase, rather than a decrease, in the incidence of cardiovascular events.

Materials and methods: We reviewed evidence from studies published in peer-reviewed journals indexed in Medline, EMBASE and CINAHL that compared different BP goals.

Results: Post-hoc analyses of randomized trials specifically conducted to test the hypothesis of the "J-shaped curve" yielded conflicting results. However, trials directly comparing different blood pressure goals and meta-analyses showed that in-treatment blood pressure values below the usual goal of less than $140 / 90 \mathrm{mmHg}$ improve outcomes in patients at increased vascular risk. Discussion: The fear that an excessive reduction in blood pressure may be dangerous is inconsistent with the available data and probably conditioned by the adverse impact of other risk factors that may be more frequent in patients with low values of achieved blood pressure. The association between blood pressure reduction and cardiovascular risk seems to be linear and not J-shaped. (c) 2012 Elsevier Srl. All rights reserved.
\end{abstract}

\footnotetext{
* Corresponding author: Section of Cardiology, Hospital "Media Valle del Tevere", ASL 2 Umbria, Perugia, Italy. E-mail: fangeli@cardionet.it (F. Angeli).
} 


\section{Introduction}

Hypertension is a major risk factor for cardiovascular (CV) and cerebrovascular disease. It is more common than other $\mathrm{CV}$ risk factors such as cigarette smoking, dyslipidemia, and diabetes $[1,2]$. In addition to the direct association between blood pressure (BP) levels and the risk of CV disease, the beneficial effect of BP reduction in controlling CV risk is well documented [3-5]. Epidemiological observations suggested that lower BP values, even within the normotensive range, are protective against future vascular events [6,7]. Large randomized controlled trials (RCTs) conducted in mixed populations of normotensive and hypertensive patients with coronary artery disease $[8,9,10]$ (CAD) or high vascular risk [11] showed, with one exception [10], the effectiveness of BP lowering, even in the normotensive range, for reducing vascular risk. However, some retrospective analyses of major trials in patients with CAD or high vascular risk [12] suggested worse outcomes not only in association with high BP values, but also with low achieved BP levels, thus raising the hypothesis of the J-shaped curve paradox [13].

The present review focuses on the "J-shaped curve" hypothesis using retrospective and initial prospective studies and tries to summarize the current state of evidence regarding optimal BP goals in patients with high vascular risk.

\section{The "J-Curve" hypothesis: an artifact?}

After the pioneering observations by Anderson [14], Stewart [15] and Cruickshank [13], subsequent studies confirmed the occurrence of an increase, rather than a decrease, in cardiac morbidity and mortality in patients with low values of achieved diastolic BP $[12,16,17]$.

The International Verapamil-Trandolapril Study (INVEST), a randomized study that included hypertensive patients with $C A D$, showed a J-shaped relationship between the levels of achieved diastolic BP and the risk of $\mathrm{MI}$ (and, to a much lesser extent, that of stroke) [17]. The risk of the primary outcome of the study (all-cause death, non-fatal MI, non-fatal stroke) showed a J-shaped relationship with both diastolic and systolic BP [17], the latter being unexpected on the basis of pathophysiology. Similar results were obtained by the Valsartan Antihypertensive Long-Term Use Evaluation (VALUE) [16] trial and by the Ongoing Telmisartan Alone and in Combination with Ramipril Global Endpoint Trial (ONTARGET) [12] (Fig. 1). In addition, a post-hoc analysis of the Hypertension Optimal Treatment (HOT) study suggested that the relationship between $\mathrm{MI}$ and achieved diastolic BP was $\mathrm{J}$-shaped in the subset with CAD at entry, but not in the subset without CAD.

In a pooled analysis of 13 studies conducted in more than 48,000 patients with treated hypertension, the risk of cardiac events increased in patients with low values of treated diastolic BP, whereas the risk of stroke did not show any $\mathrm{J}$-shaped relation with diastolic BP [18].

Caveats in interpreting the J-curve. In his review article, Marschner showed that the evaluation of the prognostic value of a primary risk factor (in our case, BP) may be easily confounded by the association with other (i.e., residual) risk factors [19]. Such a situation may occur when a study is conducted on patients carrying, by protocol, one or more

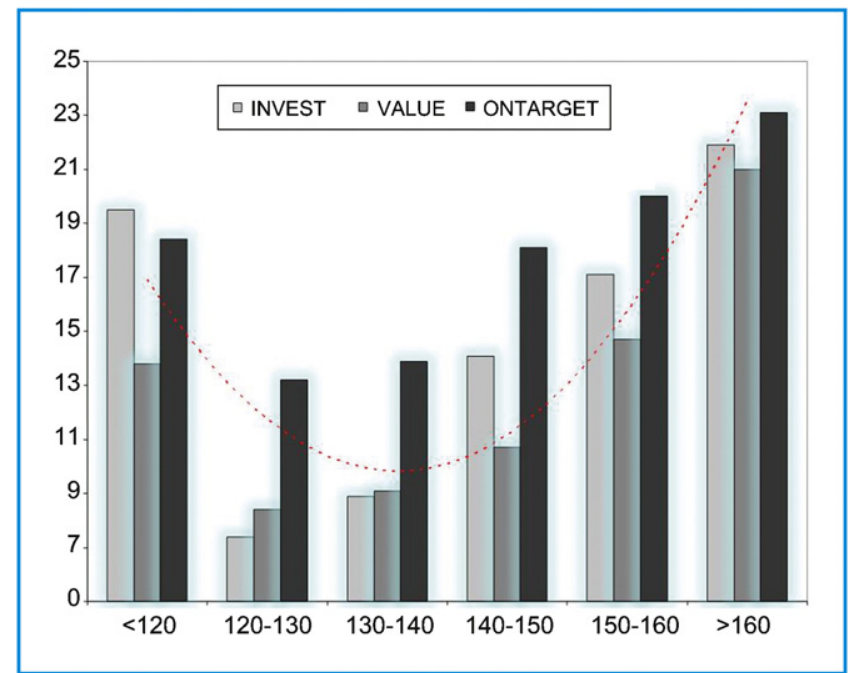

Figure 1 The J-curve phenomenon suggested by three recent clinical trials. The figure depicts the possible association between systolic blood pressure and cardiovascular events.

prognostically important risk factors. In such a context, patients with a less phenotypic expression of the primary risk factor (in our case, with low values of BP) may have an enhanced expression of other risk factors. Such a condition may have occurred, for example, in the aforementioned analysis of the INVEST study, in which the patients with low achieved diastolic BP were older and also had a higher incidence of prior myocardial infarction (MI), cancer, heart failure (HF), and diabetes compared to those with high diastolic BP [17]. Pulse pressure (PP), a prognostically important risk factor, was also higher in patients with low diastolic $\mathrm{BP}$ [17]. This was also the case in the ONTARGET trial, where the patients with lower achieved $\mathrm{BP}$ also had higher incidences of CAD and previous MI [12].

Therefore, if one combines the prognostic impact of residual risk factors (prior $\mathrm{MI}, \mathrm{HF}$, cancer, etc.) with that of the primary risk factor (BP), the aggregate effect is a nonlinear distortion of the otherwise linear relationship between the primary risk factor and the outcome. Such a distortion may easily produce an apparent threshold, or $\mathrm{J}$-curve relationship, even if the true underlying relationship is linear.

In summary, patients with low BP may be at higher risk of adverse events not because of the "excessive reduction of BP" but because of the more frequent coexistence with other, prognostically important, residual risk factors. In other words, this may be a good context for the concept of "reverse causality" (e.g., residual risk factors being the primary cause of both the low BP values and the increased risk of events).

\section{BP lowering in the normotensive range: lessons from RCTs}

Active treatment vs. placebo. Some placebo-controlled trials evaluated the hypothesis that active anti-hypertensive treatment might have a direct and clinically significant CV 
benefit in patients with a mean baseline BP less than $140 / 90 \mathrm{mmHg}$. Some, but not all, of these trials demonstrated benefit from active therapy. Three RCTs demonstrated benefit in term of CV outcome. The first of these trials, the Heart Outcomes Protection Evaluation (HOPE) trial [11], clearly demonstrated a beneficial effect of ACEinhibitors (ACE-Is) in patients at high risk of vascular disease as a consequence of $C A D$, previous stroke, peripheral arterial disease or complicated diabetes. The average BP at entry in these patients was only $139 / 79 \mathrm{mmHg}$. In these patients, ramipril significantly prevented CV death, stroke, MI, HF and diabetic microvascular complications including nephropathy compared to placebo. In addition, it reduced the need for angioplasty and bypass surgery [11].

The second study, the European Trial on Reduction of Cardiac Events with Perindopril in Stable Coronary Artery Disease (EUROPA) [8] analyzed the prognostic impact of perindopril in over 10,000 patients with CAD. The mean BP at entry was $137 / 82 \mathrm{mmHg}$, and only 27 percent had a history of hypertension. The patients were randomly assigned to perindopril ( $8 \mathrm{mg}$ once daily) or placebo. The EUROPA trial showed that the addition of the ACE-I perindopril to standard therapy significantly reduced the composite end point of $\mathrm{CV}$ death, MI, and cardiac arrest. There was at least a trend toward a similar benefit in each of the components of the primary endpoint, and the effect was consistent in all predefined subgroups. Achieved BP in the perindopril group averaged $128 / 78 \mathrm{mmHg}$, a value that was $5 / 2 \mathrm{mmHg}$ lower on average than seen with placebo.

In the Comparison of Amlodipine vs. Enalapril to Limit Occurrences of Thrombosis (CAMELOT) trial [9], active treatment with amlodipine or enalapril was compared with placebo in 1,991 patients with known CAD. The mean baseline BP was $129 / 78 \mathrm{mmHg}$, and both amlodipine and enalapril produced greater average reductions in BP than placebo $(4.8 / 2.5$ and $4.9 / 2.4$ versus $0.7 / 0.6 \mathrm{mmHg})$. Although there was a non-significant difference in the rate of "hard" CV endpoints (all-cause mortality, nonfatal MI, or stroke) with amlodipine or enalapril compared to placebo, some components of the primary end-point (coronary revascularization and hospitalization for angina) were significantly reduced by amlodipine compared to enalapril and placebo [9].

Other trials showed no benefit from active therapy compared to placebo even though lower BPs were attained. In particular, the Prevention of Events with Angiotensin Converting Enzyme Inhibition (PEACE) trial [10] showed that the addition of trandolapril to standard therapy failed to provide any benefit in terms of death from CV causes, MI, or coronary revascularization (primary end-point). None of the examined subgroups benefited from ACE-I therapy. The PEACE trial [10] targeted patients with known CAD and ejection fractions (EF) equal to or greater than $40 \%$ and was specifically conceived to extend the observations that emerged in the HOPE study to a lower risk population. Notably, the mean baseline BP in the PEACE trial was $133 / 78 \mathrm{mmHg}$. However, the patients enrolled in the PEACE trial were at lower CV risk and were more likely to have been treated with beta-blockers, antiplatelet drugs and lipid-lowering therapy than the HOPE [11] and EUROPA cohorts [8].

The Telmisartan Randomised AssessmeNt Study in ACE iNtolerant subjects with cardiovascular Disease (TRANSCEND) trial [20] randomly assigned high-risk patients who were similar to those in HOPE [11] but did not tolerate ACE-Is to either telmisartan $80 \mathrm{mg} /$ day or placebo. The mean baseline BP was $141 / 82 \mathrm{mmHg}$. At a median follow-up of 56 months, the mean BP was $4.0 / 2.2 \mathrm{mmHg}$ lower in the telmisartan group than in the placebo group. Despite this gradient in achieved BP, there was no statistically significant difference between the two groups in the primary composite outcome of CV death, MI, stroke, or hospitalization for HF. Again, the use of statins, beta blockers, and antiplatelet agents was much greater in TRANSCEND than in HOPE. Furthermore, the achieved BP gradient between the two arms may not have been enough to guarantee a prognostic benefit [21].

Two other trials showed no benefit of active treatment compared to placebo despite a greater BP reduction in the active treatment group. In the Nateglinide and Valsartan in Impaired Glucose Tolerance Outcomes Research (NAVIGATOR) trial [22], patients with impaired glucose tolerance and either established CV disease or one or more risk factors for CV disease were randomly assigned to valsartan or placebo. The mean BP at baseline was 140/83 mmHg, and after a median follow-up of five years, the mean BP decreased significantly more in the valsartan group (6.3/4.4 versus $3.8 / 3.0 \mathrm{mmHg}$ ). No significant differences were observed in the incidence of a composite outcome of death from CV causes, nonfatal MI, nonfatal stroke, hospitalization for HF, arterial revascularization, or hospitalization for unstable angina.

In the A Coronary disease Trial Investigating Outcome with Nifedipine GITS (ACTION) trial [23], 7,665 patients with chronic stable angina were randomly assigned to long-acting nifedipine or placebo. Active treatment had no effect on the incidence of the primary end-point (major CV events, including death by any cause, acute $\mathrm{Ml}$, refractory angina, new overt HF, debilitating stroke, and peripheral revascularization). The mean baseline BP was $137 / 80 \mathrm{mmHg}$ and was significantly lower in the nifedipine group at end the end of the study $(130 / 75$ versus $136 / 78 \mathrm{mmHg})$.

RCTs comparing different BP goals. Only two trials $[24,25]$ have directly compared BP goals to test the hypothesis that lower attained BPs (below the usual goal of less than $140 / 90 \mathrm{mmHg}$ ) improve outcomes in patients at increased vascular risk.

In the Studio Italiano Sugli Effetti Cardiovascolari del Controllo della Pressione Arteriosa Sistolica (Cardio-Sis) [24], 1,111 treated non-diabetic high risk patients were randomly assigned to a goal systolic BP of $<140 \mathrm{mmHg}$ (usual control) or $<130 \mathrm{mmHg}$ (tight control). Open-label agents were used to reach the randomized BP goals. The primary study end-point was the proportion of patients with new development or lack of regression of electrocardiographic left ventricular hypertrophy $(\mathrm{LVH}) 2$ years after randomization, and the main secondary end-point was a composite pool of pre-specified CV events and death. The primary end-point of the study occurred less frequently in the tight than in the usual control group (odds ratio 0.63; $95 \%$ confidence interval [Cl] 0.43-0.91; $\mathrm{p}=0.013$ ). In addition, the secondary endpoint occurred less frequently in the tight than in the usual control group (hazard ratio $0.50 ; \mathrm{Cl} 0.31-0.79 ; \mathrm{p}=0.003$ ) (Fig. 2). A pre-specified subgroup analysis did not show any interaction between the subsets with and without overt CV disease at entry in the risk of both the primary and secondary end-points. 


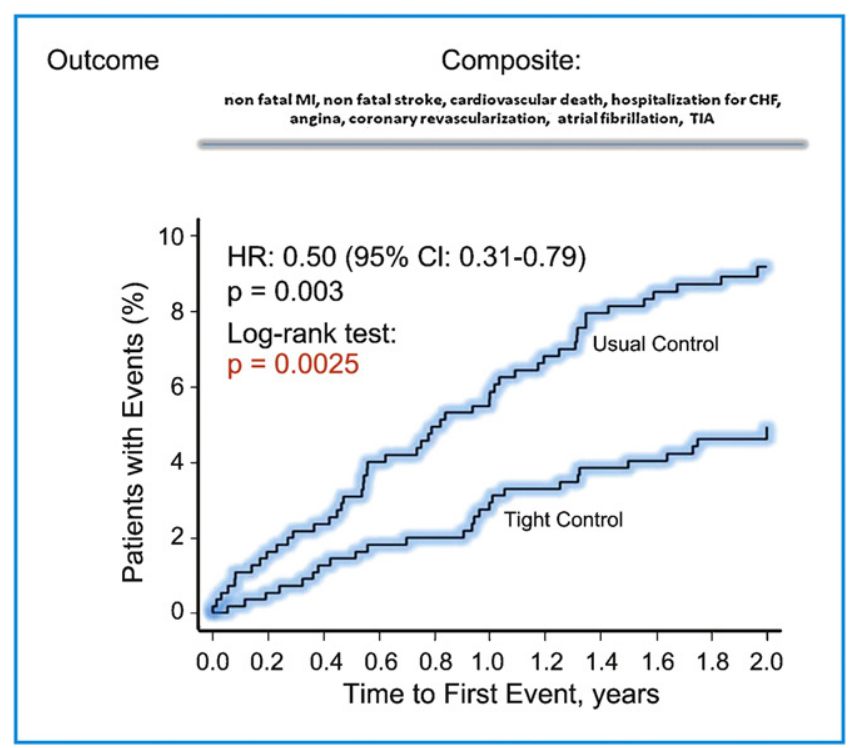

Figure 2 Effect of tight blood pressure control on cardiovascular events in hypertensive patients at high vascular risk.

The Action to Control Cardiovascular Risk in Diabetes (ACCORD BP) trial [25] randomly assigned 4,733 patients with type II diabetes mellitus with established CV disease or at least two additional risk factors to systolic BP targets of either less than $120 \mathrm{mmHg}$ or less than $140 \mathrm{mmHg}$. After a mean follow-up of 4.7 years, there was no significant difference in the annual rate of the primary endpoint (composite of nonfatal MI, nonfatal stroke, or death from CV causes; hazard ratio $0.88,95 \% \mathrm{Cl} 0.73-1.06)$. However, patients randomized to a goal BP of less than $120 \mathrm{mmHg}$ showed a significantly lower annual rate of stroke $(0.32$ versus 0.53 percent/year, hazard ratio $0.59,95 \% \mathrm{Cl} 0.39-0.89)$, with 89 patients that needed to be treated to prevent one stroke in five years.

Observations from aggregate analyses. Both the Blood Pressure Lowering Treatment Trialists' Collaboration (BPLTTC) $[26,27]$ and Staessen et al. [4] have reported, through metaregression analyses of intervention trials, an association between systolic BP reduction and the reduced risk of total $\mathrm{CV}$ events. In meta-regression analyses, we also confirmed the relationship between the degree of $\mathrm{BP}$ reduction and the protection from stroke and congestive HF, whereas the protection from $\mathrm{MI}$ did not increase appreciably with a more aggressive BP control [5,28,29]. In particular, the risk of congestive HF decreased by $24 \%$ for each $5 \mathrm{mmHg}$ reduction in systolic BP [5].

More recently, we conducted a meta-analysis of trials including 221,024 patients that compared different BPlowering agents with placebo or active treatments in patients with hypertension or composite features of high CV risk. The outcome measure was a composite cardiovascular endpoint (CCEP) including MI, stroke, CV death and congestive HF. In a multivariable meta-regression analysis, for each $5-\mathrm{mmHg}$ reduction in systolic BP, there was $13 \%$ less risk of CCEP (95\% Cl: $8-19, \mathrm{p}=0.001)$ and, for each $2-\mathrm{mmHg}$ reduction in diastolic BP, there was $12 \%$ less risk of CCEP $(95 \% \mathrm{Cl}: 7-16$, $\mathrm{p}=0.001$ ).

Notably, such associations were linear and not J-shaped [30]. The lack of any evidence for the J-curve phenomenon in the context of meta-regression analyses is particularly important. Indeed, any potential confounder or residual risk factor tends to be equally distributed by randomization between the treatment groups and is therefore unlikely to bias the results, as may occur, for example, in analyses based solely on different levels of achieved BP.

\section{Goal blood pressure: recommendations in clinical practice}

The standard goal of antihypertensive therapy [31,32] (i.e., less than $140 / 90 \mathrm{mmHg}$ ) is well established and should be maintained. However, we should not ignore the fact that (a) most patients with high vascular risk due to $C A D$, stroke or diabetes continue to have poorly controlled BP; (b) this situation has not changed over the past 15 years. The optimal $\mathrm{BP}$ target in these patients remains undefined. Lower BP values have been associated with a lower risk of both stroke and cardiac complications.

However, the fear that an excessive reduction of diastolic $\mathrm{BP}$ may be dangerous (the J-curve phenomenon) is inconsistent with our analysis and probably conditioned by the adverse impact of other risk factors that may be more frequent in patients with low values of achieved BP. Consequently, a reasonable $\mathrm{BP}$ target to be achieved in patients with CAD appears to be in the range of $130-140 / 80-90 \mathrm{mmHg}$. Any further reduction may be safe but is perhaps not productive from a prognostic standpoint. It is of the utmost importance that BP be lowered slowly in patients with occlusive CAD. Particular caution is needed in these patients to detect early signs or symptoms of ischemia when treated diastolic BP falls below $60 \mathrm{~mm} \mathrm{Hg}$.

Given the many unanswered questions in this area, outcome-based studies specifically designed to compare different BP goals in their prognostic impacts in patients with CAD or other high-risk conditions are very much needed.

\section{Acknowledgements}

This study was funded in part by the Fondazione Umbra Cuore e Ipertensione - ONLUS, Perugia, Italy.

\section{Funding}

None of the authors of this study has financial or other reasons that could lead to a conflict of interest.

\section{References}

[1] Collins R, Peto R, MacMahon S, Hebert P, Fiebach $\mathrm{NH}$, Eberlein $\mathrm{KA}$, et al. Blood pressure, stroke, and coronary heart disease. Part 2, short-term reductions in blood pressure: Overview of randomised drug trials in their epidemiological context. Lancet 1990;335:827-38.

[2] MacMahon S, Peto R, Cutler J, Collins R, Sorlie P, Neaton J, et al. Blood pressure, stroke, and coronary heart disease. Part 1, prolonged differences in blood pressure: Prospective observational studies corrected for the regression dilution bias. Lancet 1990;335:765-74.

[3] Angeli F, Verdecchia P, Reboldi GP, Gattobigio R, Bentivoglio M, Staessen JA, et al. Calcium channel blockade to prevent stroke 
in hypertension: A meta-analysis of 13 studies with 103,793 subjects. Am J Hypertens 2004;17:817-22.

[4] Staessen JA, Wang JG, Thijs L. Cardiovascular protection and blood pressure reduction: A meta-analysis. Lancet 2001;358: 1305-15.

[5] Verdecchia P, Angeli F, Cavallini C, Gattobigio R, Gentile G, Staessen JA, et al. Blood pressure reduction and reninangiotensin system inhibition for prevention of congestive heart failure: A meta-analysis. Eur Heart J 2009;30:679-88.

[6] PROGRESS Collaborative Group. Randomised trial of a perindopril-based blood-pressure-lowering regimen among 6,105 individuals with previous stroke or transient ischaemic attack. Lancet 2001;358:1033-41.

[7] Lewington S, Clarke R, Qizilbash N, Peto R, Collins R. Agespecific relevance of usual blood pressure to vascular mortality: A meta-analysis of individual data for one million adults in 61 prospective studies. Lancet 2002;360:1903-13.

[8] Fox KM. Efficacy of perindopril in reduction of cardiovascular events among patients with stable coronary artery disease: Randomised, double-blind, placebo-controlled, multicentre trial (the europa study). Lancet 2003;362:782-8.

[9] Nissen SE, Tuzcu EM, Libby P, Thompson PD, Ghali M, Garza D, et al. Effect of antihypertensive agents on cardiovascular events in patients with coronary disease and normal blood pressure: The camelot study: A randomized controlled trial. JAMA 2004;292:2217-25.

[10] Braunwald E, Domanski MJ, Fowler SE, Geller NL, Gersh BJ, Hsia J, et al. Angiotensin-converting-enzyme inhibition in stable coronary artery disease. N Engl J Med 2004;351:2058-68.

[11] Yusuf S, Sleight P, Pogue J, Bosch J, Davies R, Dagenais G. Effects of an angiotensin-converting-enzyme inhibitor, ramipril, on cardiovascular events in high-risk patients. The heart outcomes prevention evaluation study investigators. N Engl J Med 2000;342:145-53.

[12] Sleight P, Redon J, Verdecchia P, Mancia G, Gao P, Fagard R, et al. Prognostic value of blood pressure in patients with high vascular risk in the ongoing telmisartan alone and in combination with ramipril global endpoint trial study. J Hypertens 2009;27:1360-9.

[13] Cruickshank JM, Thorp JM, Zacharias FJ. Benefits and potential harm of lowering high blood pressure. Lancet 1987;1:581-4.

[14] Anderson TW. Re-examination of some of the framingham blood-pressure data. Lancet 1978;2:1139-41.

[15] Stewart IM. Relation of reduction in pressure to first myocardial infarction in patients receiving treatment for severe hypertension. Lancet 1979;1:861-5.

[16] Julius S, Kjeldsen SE, Weber M, Brunner HR, Ekman S, Hansson L, et al. Outcomes in hypertensive patients at high cardiovascular risk treated with regimens based on valsartan or amlodipine: The value randomised trial. Lancet 2004; 363:2022-31.

[17] Messerli FH, Mancia G, Conti CR, Hewkin AC, Kupfer S, Champion A, et al. Dogma disputed: Can aggressively lowering blood pressure in hypertensive patients with coronary artery disease be dangerous? Ann Intern Med 2006;144:884-93.

[18] Farnett L, Mulrow CD, Linn WD, Lucey CR, Tuley MR. The j-curve phenomenon and the treatment of hypertension. Is there a point beyond which pressure reduction is dangerous? JAMA 1991;265:489-95.
[19] Marschner IC, Simes RJ, Keech A. Biases in the identification of risk factor thresholds and j-curves. Am J Epidemiol 2007; 166:824-31.

[20] Yusuf S, Teo K, Anderson C, Pogue J, Dyal L, Copland I, et al. Effects of the angiotensin-receptor blocker telmisartan on cardiovascular events in high-risk patients intolerant to angiotensin-converting enzyme inhibitors: A randomised controlled trial. Lancet 2008;372:1174-83.

[21] Verdecchia P, Gentile G, Angeli F, Mazzotta G, Mancia G, Reboldi G. Influence of blood pressure reduction on composite cardiovascular endpoints in clinical trials. J Hypertens 2010; 28:1356-65.

[22] McMurray JJ, Holman RR, Haffner SM, Bethel MA, Holzhauer B, Hua TA, et al. Effect of valsartan on the incidence of diabetes and cardiovascular events. N Engl J Med 2010;362:1477-90.

[23] Poole-Wilson PA, Lubsen J, Kirwan BA, van Dalen FJ, Wagener G, Danchin $\mathrm{N}$, et al. Effect of long-acting nifedipine on mortality and cardiovascular morbidity in patients with stable angina requiring treatment (action trial): Randomised controlled trial. Lancet 2004;364:849-57.

[24] Verdecchia P, Staessen JA, Angeli F, de Simone G, Achilli A, Ganau A, et al. Usual versus tight control of systolic blood pressure in non-diabetic patients with hypertension (cardiosis): An open-label randomised trial. Lancet 2009;374: 525-33.

[25] Cushman WC, Evans GW, Byington RP, Goff Jr DC, Grimm Jr RH, Cutler JA, et al. Effects of intensive blood-pressure control in type 2 diabetes mellitus. N Engl J Med 2010;362:1575-85.

[26] Turnbull F, Neal B, Ninomiya T, Algert C, Arima H, Barzi F, et al. Effects of different regimens to lower blood pressure on major cardiovascular events in older and younger adults: Meta-analysis of randomised trials. BMJ 2008;336:1121-3.

[27] Turnbull F. Effects of different blood-pressure-lowering regimens on major cardiovascular events: Results of prospectively-designed overviews of randomised trials. Lancet 2003; 362:1527-35.

[28] Reboldi G, Gentile G, Angeli F, Ambrosio G, Mancia G, Verdecchia P. Effects of intensive blood pressure reduction on myocardial infarction and stroke in diabetes: A meta-analysis in 73,913 patients. J Hypertens 2011;29:1253-69.

[29] Verdecchia P, Reboldi G, Angeli F, Gattobigio R, Bentivoglio M, Thijs $L$, et al. Angiotensin-converting enzyme inhibitors and calcium channel blockers for coronary heart disease and stroke prevention. Hypertension 2005;46:386-92.

[30] Verdecchia P, Gentile G, Angeli F, Mazzotta G, Mancia G, Reboldi G. Influence of blood pressure reduction on composite cardiovascular endpoints in clinical trials. J Hypertens 2010;28:1356-65.

[31] Mancia G, De Backer G, Dominiczak A, Cifkova R, Fagard R, Germano G, et al. 2007 guidelines for the management of arterial hypertension: The task force for the management of arterial hypertension of the european society of hypertension (esh) and of the european society of cardiology (esc). J Hypertens 2007;25:1105-87.

[32] Chobanian AV, Bakris GL, Black HR, Cushman WC, Green LA, Izzo Jr. JL, et al. The seventh report of the joint national committee on prevention, detection, evaluation, and treatment of high blood pressure: The jnc 7 report. JAMA 2003; 289:2560-72. 